\title{
ROCKY SHORELINE EXTRACTION USING A DEEP LEARNING MODEL AND OBJECT-BASED IMAGE ANALYSIS
}

\author{
S. Bengoufa ${ }^{1,2,3 *}$, S. Niculescu ${ }^{1}$, M.K. Mihoubi ${ }^{3}$, R. Belkessa ${ }^{2}$, K. Abbad ${ }^{2}$ \\ 1 University of Western Brittany, CNRS, LETG Brest UMR 6554 CNRS, Technopôle Brest-Iroise, Plouzané-Brest, 29280, France- \\ bengoufasoumia@gmail.com_Simona.Niculescu@univ-brest.fr \\ 2 Ecole Nationale Supérieure des Sciences de la Mer et de l'Aménagement du Littoral (ENSSMAL), 16320 Algiers, Algeria- \\ belkessa.r.littoral@gmail.com_abbad.katia@gmail.com \\ 3 Ecole National Supérieure de l'Hydraulique, Laboratoire de Mobilisation et de Valorisation des Ressources en Eau (MVRE), \\ 09000 BLIDA, Algeria- mihkam@ensh.dz
}

\section{Commission III, WG III/1}

KEY WORDS: Convolutional neural network (CNN), Object-Based Image Analysis (OBIA), Shoreline detection, Marine Lichen.

\begin{abstract}
:
In the context of the increasing anthropogenic influence on the coastal areas that are subject to high climate variability, the main challenge is to understand its current dynamics and to predict its future evolution. Therefore, monitoring of the shoreline kinematics is a key factor for the coastal erosion assessment and an essential feature for the sustainable management of these naturally vulnerable areas.

This work focuses on the detection and extraction of the shoreline, basing on a specific remote sensing methodology using Very High Resolution (VHR) optical images. Indeed, an integrated approach based on a Deep Learning model, which is the Convolutional Neural Network (CNN) and Object Based Image Analysis (OBIA) has been developed. This study aims to evaluate the methodological contribution of this integrated approach for the (semi)-automatic extraction of the rocky shoreline, for which the botanical indicator has been chosen. Therefore the upper limit of black marine lichen has been detected and extracted as the target shoreline. It is the first indication of a (semi)-automatic detection of such a complex type of shoreline.

The classification results derived from the combined CNN model and OBIA methods had achieved a high overall accuracy of 0.94 . The extracted shoreline have been compared to a shoreline of reference derived from a traditional method that is a manual digitizing. The distances between the two shorelines has been calculated in order to assess the accuracy of the extraction method. This comparison revealed that $76 \%$ of the extracted shoreline lies within $1 \mathrm{~m}$, and $35 \%$ lies within $0.5 \mathrm{~m}$ of reference one. Therefore, the CNN model integrated to OBIA was successfully shown to be a good method for shoreline extraction and could offer an immediate insight regarding rocky shoreline position, providing an alternative to its monitoring.
\end{abstract}

\section{INTRODUCTION}

Significant investments are being made to assess and mitigate the risks that coastal erosion hazard pose to the public and ecosystem services (Westoby et al., 2018). This hazard is expressed by the retreat of the shoreline, which is a key indicator of erosion risk assessment. Therefore, coastal hazard monitoring acquires data acquisition related to the shoreline, more or less frequently, and over large area, with the main purpose of analyzing and informing on the shoreline kinematic and the coastal environment changes.

The shoreline monitoring by traditional techniques is often a tedious and subjective task (Boak and Turner, 2005), indeed, automatic and reproducible techniques are needed (Bagli and Soille, 2003).
In fact, an improved (semi)-automatic detection of the shoreline via remote sensed images processing could significantly optimize the time and costs, and reduce the subjective component of manual shoreline digitization. In this regard, many different research fields could benefit from this improved shoreline extraction, such as coastal vulnerability and erosion assessment, environmental risk analysis, land use planning, and coastal management and engineering (Bengoufa, et al. 2021).

Recently, shoreline detection has received considerable attention basing on remotely sensed images processing. However, there is a multitude of choices of shoreline indicators, depending on the geomorphological type of the coastline. This variability is often not taken into consideration in previous shoreline detection studies, which generally consider the landsea boundary as a shoreline, or only focus on the accumulation coastline so-called sandy coasts. Thus, their developed methods

\footnotetext{
* Corresponding author
} 
are often unsuitable for more complex coastline types such as the rocky coasts (Mallet et al. 2012).

With the development of Very High-Resolution (VHR) and multispectral sensors, detailed mapping of the coastal land cover become possible. Recently several Machine Learning models (Random Forest, SVM, K-NN, etc.) have been successfully adopted for remote sensing applications (Niculescu et al., 2018) and land cover detection. In fact, with the DeepLearning (DL) methods, computational models composed of multiple processing layers can learn data representations with multiple levels of abstraction (LeCun et al., 2015).

Therefore, DL methods, in particular Convolutional Neural Network (CNN), have achieved success for classification of Remote Sensed images (Zhu et al., 2017). Generally CNN integrates texture and spatial context of images in the analysis process on the pixel level. However, this cannot accurately detect object borders (Ghorbanzadeh et al., 2021). Thus, Object Based Image Analysis (OBIA) provides the ability to integrate shape, texture, and size parameters, as well as other topological features into the analysis process, along with the spectral information of the pixels.

In this context of the lack of studies proposing detection methods of the rocky shoreline, this work attempts to use an integrated approach of CNN model and OBIA for automatic shoreline extraction from multispectral VHR images.

\section{STUDY AREA}

The case study is a subset of Mostaganem Province Coastline (west of Algeria) (Figure1), which has an interesting landscape and geomorphological diversity giving it touristic potential.

The western part of this coastline is highly artificialized and built-up. Hence, there is a need for the implementation of a roadmap to control and predict the harmful effects of anthropogenic activities on the shoreline.

The case study is a western-subset pilot site of a much larger coastal zone (Figure1) selected for further long-term analysis of shoreline kinematics. This subset of a $6 \mathrm{~km}$ length of shoreline $\left(35^{\circ} 51^{\prime} 0.55^{\prime \prime} \mathrm{N} 0^{\circ} 00^{\prime} 42.98^{\prime \prime} \mathrm{E}\right)$ is a micro-tidal coast with an insignificant tidal effect characterised by low rocky cliffs.

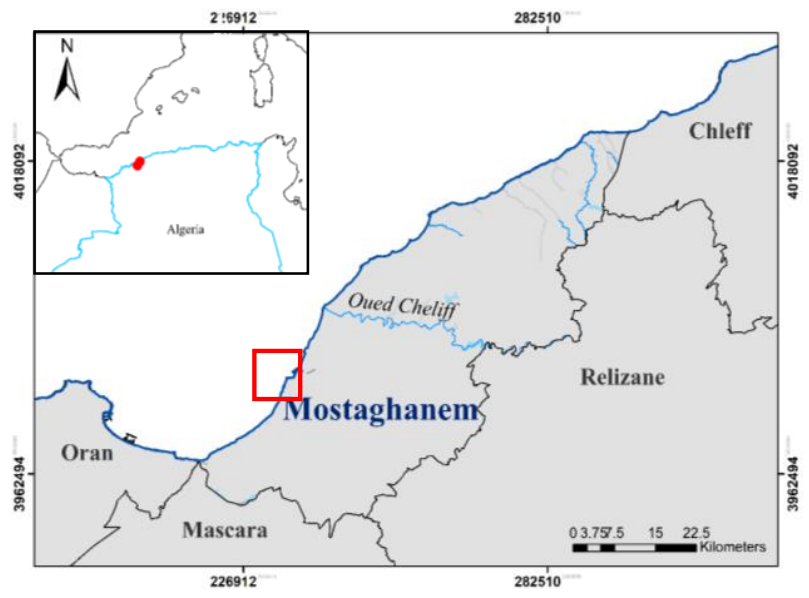

Figure 1. Geographic situation of the study area

\section{METHOD}

In this study, an integrated method of CNN model and an OBIA approach (Figure 2) was developed for the extraction of a rocky shoreline. Shoreline retreat in this geomorphological form of coast is faster in the less steep slopes and less consolidated profiles (topographic structure). Knowing that the rock types (geological structure) in rough marine areas are strongly subject to wave and swell action, the hardest rocks can become soft if they are susceptible to physical and chemical alterations.

\subsection{Identification of Shoreline Indicator}

For rocky coastline monitoring, it is necessary to define a shoreline indicator detectable on satellite images. Boak and turner, (2005) has reviewed the main shoreline indicators for rocky and cliff coasts (Bluff top/cliff top, Base of bluff/cliffs, botanical indicators, etc.). The choice of one of these indicators has to meet the major shoreline recognition requirements. Therefore, the indicator has to be recognizable on remote sensed images; continuous along the coast; and the across-shore variations in its position due to water level changes has to be at a minimum. These conditions can be met by "botanical" indicators, which are generally preferred.

Information on the botanical habitat of the rocky coast is specified in the European Nature Information System (key B3: Cliff, ledges and rocky coast) (Romero et al., 2016) (Davies, et al. 2004). The supralittoral level of this coast is characterised by the presence of marine lichen (Verrucaria amphibia, $V$. Maura), which appears as a black belt on the substrate.

In fact, the upper edge of this belt is considered a good shoreline indicator of the rocky coast (Bonnot-Courtois and Levasseur, 2002).

\subsection{Data acquisition}

For the automatic detection of the shoreline, the needs in datasets and remotely sensed images were defined depending on the geomorphological type of the studied coastline and the chosen shoreline indicator.

Therefore, on the same day as the in-situ sampling, a special programming of the acquisition Pleiades satellite images was carried out. It is Very High Resolution scenes of $0.5 \mathrm{~m} \times 0.5 \mathrm{~m}$, with 4 spectral bands: visible Red, Green, Blue and Near InfraRed (RGB and NIR).

The objective being to highlight the superior limit of black marine lichen, four classes were assigned: i) Water ii) Lichen iii) Soil, and iv) Vegetation. The sampling consisted of collecting Global Position System (GPS) points for each of the three classes except for Water class (undertaking manually on VHR image).

In-situ data were collected using GPS with centimetric precision in real-time kinematics mode. It is a relative positioning system (differential positioning) that involves two GPS receivers tracking the same satellites simultaneously to determine their relative coordinates. One receiver is mobile and the other is the reference (base) that remains stationary with a precisely known coordinate. 
This in-situ data performed the same day as the images acquisition were used for training and testing the classification model. Therefore, the samples were randomly divided into two independent datasets: $80 \%$ for training the CNN model and $20 \%$ for the accuracy evaluation (test datasets).

\subsection{Image Processing}

The remotely sensed images were processed basing on the CNN model. It is a deep learning model inspired from the architectures of biological neural networks. Therefore, $\mathrm{CNN}$ is a multi-layer feed-forward neural network model where the layers are interconnected to each other by a set of learnable weights and biases (Romero et al., 2016).

The convolutional layers use, as inputs, image sample patches that capture different features by scanning the entire image. The principle is based on the generation of feature maps from each layer using the image patches in order to feed the subsequent layers (Figure 3).

The main goal of this work is to purpose a shoreline extraction method which incorporates the image object to a CNN model. Therefore the model, here developed, have quite a simple and flexible architecture. The CNN model was designed and trained in eCognition software, using 4 spectral bands (RGB and NIR) of the Pleiades images.

The training dataset was used to create convolutions input sample patches selected out of $1204 \times 1204$ pixels of the original image. To choose an adequate sample patch size we have taken under consideration the target class dimensions (Lichen class), which is a relatively narrow band. Therefore several sample sizes have been tested $(16 \times 16,10 \times 10,8 \times 8$, $4 \times 4)$.

After a cross-validation process, our designed CNN was fed by the sample size patches of $4 \times 4$. Considering this small size, the number of hidden layers was tuned to 2 (Figure 3 ) with a kernel size of $3 \times 3$ and $1 \times 1$ respectively. One max-pooling layer with a kernel size of $2 \times 2$ was used after the first convolution layer. The role of max-pooling is to merge semantically similar features. It is a non-linear top-down sampling technique that uses the maximum value of each cluster of the previous layer in order to reduce the information loss to the next convolution layers.

Furthermore, a patch size of 50 and 5000 training steps reached a good performance. A cross-validation process revealed that the optimal learning rate could be set at 0.0006. Indeed, an inadequate learning rate can either increase the time of the learning process (at a low rate), which could block the network in local minima, or decrease it (at a high rate), but the network might not reach the minima and thus in both cases it is possible to obtain incorrect weights.

The CNN model result was used as input data for the Geographic Object Based Image Analysis (GEOBIA) knowledge-based classification. GEOBIA is devoted to the development of automated methods for segmenting geospatial imagery into meaningful image objects, and valuing their characteristics across spectral spatial, and temporal scales.
The GEOBIA consists of two main steps: a) image segmentation, and b) classification.

For the Image segmentation, a Multi-Resolution (MRS) algorithm (Baatz and Schaepe, 2000), was used. MRS is known to be a popular segmentation algorithm for VHR remote sensing applications. It is based on the fractal net evolution approach, aggregating individual pixels into objects of increasing size at multiple levels in an iterative process. The critical problems of MRS are the optimization of the parameters and the evaluation of segmentation quality.

This process is based on three parameters that are tuned by the user: a) scale, which is defined as the maximum permitted heterogeneity within objects; b) shape, which is a weight between spectral and a shape factor; and c) compactness, which is a regularisation factor. After cross-validation, the Scale, Shape and Compactness parameters were fixed as: 10, 0.9 and 0.5 respectively. The resulting objects were trained and classified based on a combination of the neighbourhood, spatial properties and similarity in the $\mathrm{CNN}$ probability.

After final classification, the resulted polygons were converted to polyline format in order to select and thus extract the target shoreline. This latter is the polyline that separates the Lichen and the soil classes. In fact, the extraction is made by selecting the Lichen class ID in the attribute table and eliminating of all the other class polylines. The brute extracted polyline is a step raw vector, therefore, a smoothing procedure was necessary in order to reduce the noise and simplify the jagged shape related to the pixel shape. The smoothing method consists in locating the midpoint of each step of the stepped raw vector. This procedure is based on a smoothing distance fixed by users. This distance has to remove a smaller variation, capture the shoreline shape well, and the variation compared to the reference shoreline has to be narrow (García-Rubio et al., 2015). Therefore, several smoothing distances from $5 \mathrm{~m}$ to $80 \mathrm{~m}$ have been tested in order to fix an adequate one.

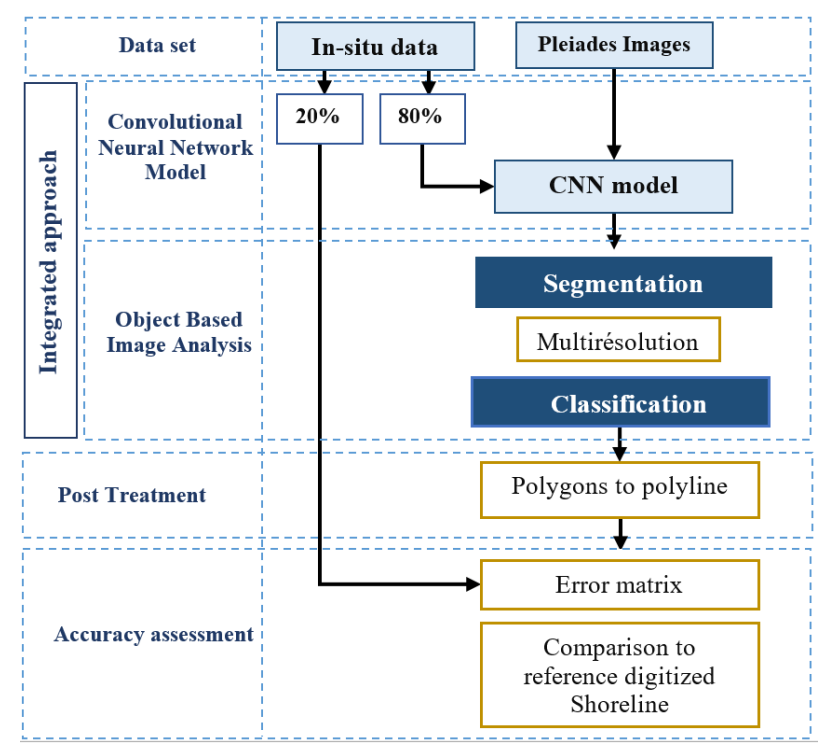

Figure 2. Methodology workflow 


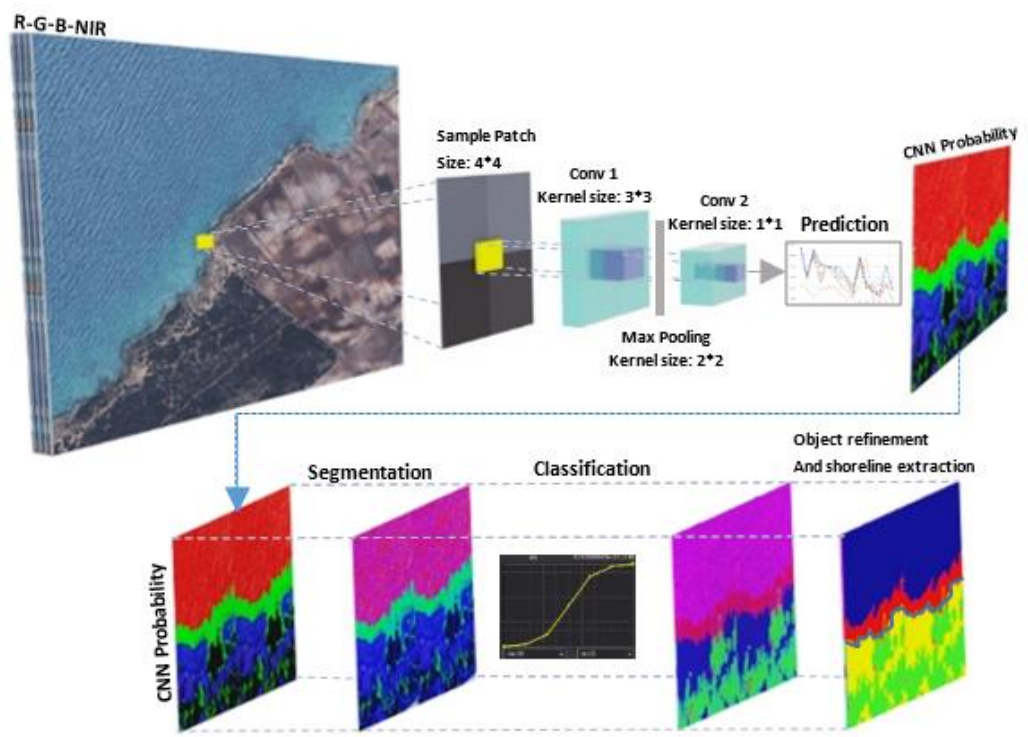

Figure 3. Integrated CNN and OBIA method workflow.

\subsection{Accuracy Assessment}

The method accuracy was assessed regarding the classification results and the extracted shoreline. This accuracy assessment allows the evaluation of the reliability and pertinence of the integrated approach, developed in this study, for rocky shoreline extraction.

Therefore, beside of the visual inspection, the accuracy of the classification results derived from the integrated approach, was assessed basing in error matrix. In fact, $20 \%$ of samples datasets that was collected during in-situ fields has been used to calculate the error matrix and the accuracy coefficients.

For the shoreline extraction accuracy, a comparison between extracted and a reference digitized shoreline was performed. Therefore, transects were generated using Digital Shoreline Analysis System (DSAS), with an equidistance of $1 \mathrm{~m}$, in order to calculate distances between the two shorelines.

\section{RESULTS AND DISCUSSION}

The results of this study were derived from the application of an integrated approach that combines a CNN model and OBIA using Pleiades optical images.

It is the first indication of such a (semi)-automatic detection of rocky shorelines. Indeed, an image classification based on a deep learning model has been undertaking using, as a training dataset, in-situ samples collected on the same day as the satellite images acquisition. The use of this dataset for the model training improved the accuracy of the results. It can be considered one of the key values of this work.

The four classes assigned for the classification process were detected (Figure 4a) with Overall Accuracy of 94\%. The detection accuracy of the target class (Black Lichen) has achieved $91 \%$, which reveals that the values of well-classified data had a high probability of being correct.

The final outcome of the combined methods was classified objects in polygon format. Since the shoreline indicator is the upper edge of the black lichen belt, a conversion of the classification results from polygons to polylines format is necessary. This edge has been selected and extracted as the target shoreline. Figure $4 \mathrm{~b}$ presents the final extracted shoreline after the conversion of polygon into polyline format.

As it has been highlighted above, an adequate smoothing distance is necessary to simplify the jagged shape of the extracted shoreline. The distance of $10 \mathrm{~m}$ resulted in shorelines with less variation, captured the shoreline shape well, and had the narrowest variation compared to the reference shoreline.

A qualitative evaluation basing on a visual inspection emphasized that the shape of the extracted shoreline is quite well corresponding to the shape of the upper limit of black Lichen observed on the remote sensed image (Figure 5)

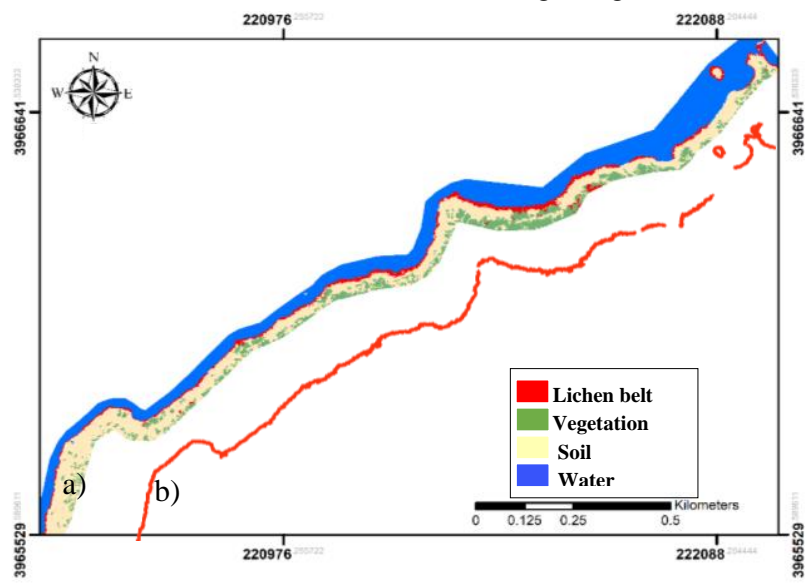

Figure 4. a) CNN-OBIA classification results, b) extracted. 

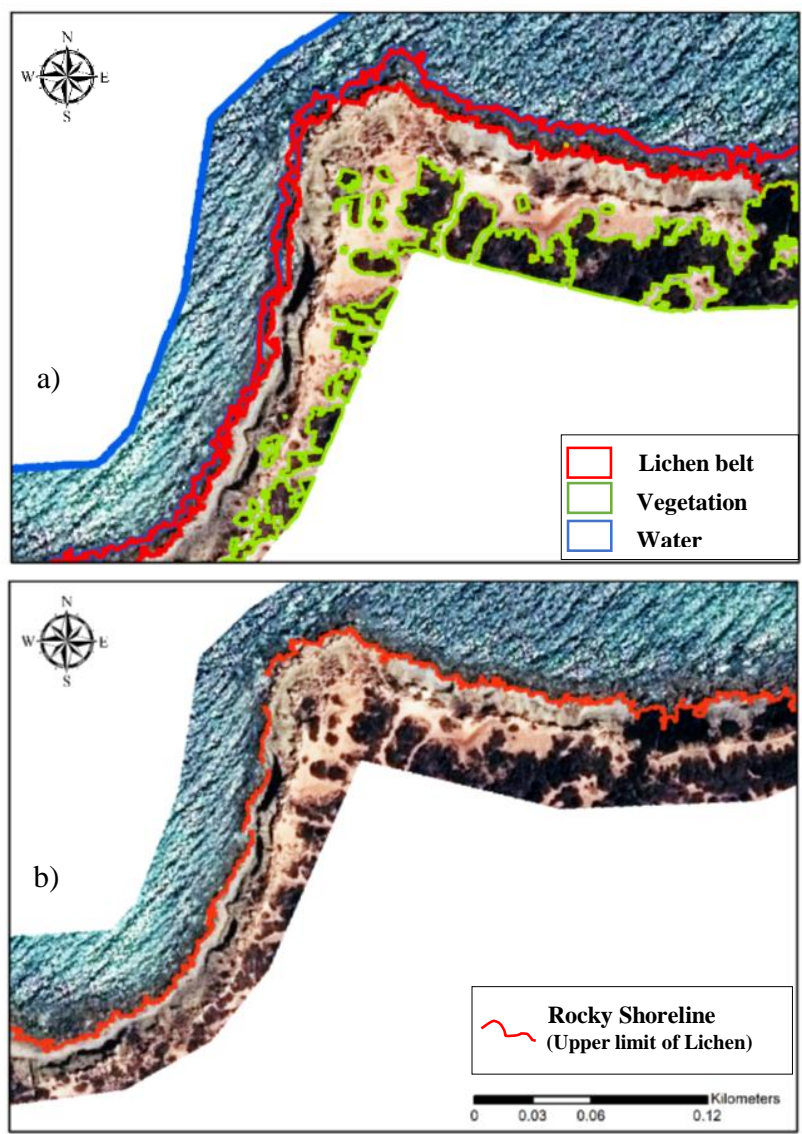

Figure 5. CNN-OBIA results. a) Classified objects b) extracted shoreline (upper limit of black Lichen)

In order to assess its reliability, the shoreline resulted from the combined approach have been compared to a reference shoreline derived from a traditional method. This method consisted in a manual digitizing of the superior limit of Lichen belt.

The quantitative assessment has been conducted by calculating the distances between the two shorelines using DSAS (Thieler et al., 2009) processed in ArcGIS software. The Net Shoreline Movement (NSM) that represent the metric distances between two shorelines are calculated. An overview of NSM (distances) is presented in Figure 6. The results reveal that $76 \%$ of the extracted shoreline lies within $1 \mathrm{~m}$, and $35 \%$ lies within $0.5 \mathrm{~m}$ of the digitized one, with maximum distance of $2,3 \mathrm{~m}$.
The highest distance values are observed in sectors where terrestrial vegetation is close to the Lichen boundary (Figure 7). Excluding these sectors, the distances demonstrate that the automatic extraction method is quite effective in detecting the rocky shoreline with the accuracy of $<1 \mathrm{~m}$ over the area taken as a reference for the analysis.

Therefore, the method has limitations when the vegetation is close to the shore, where misclassifications are committed (Figure 7). This is due to the high confusion between the terrestrial vegetation and the Lichen cover, which have similar spectral information. Post-processing using the NDVI index could resolve these classification errors.

Furthermore, the results showed that the integrated approach can, also, be used to detect the shoreline of islets (Figure 8). The very high resolution of the Pleiades images played an important role in this fine-scale detection.

The in-situ measurement stays the most relevant method for shoreline detection; however, it has an important time, logistical and human resources cost. The CNN model integrated to OBIA can offer an immediate insight regarding rocky shoreline position, providing an alternative for its monitoring.

Even though in terms of overall accuracy, the performance of ML algorithms generally outperforms -shallow- machine learning techniques (Campos-Taberner et al., 2016; Liu et al., 2018), the understanding of these algorithms and their interpretation is typically limited (Campos-Taberner et al., 2020; Montavon et al., 2018).

Therefore, the major point to consider is the difficulties of the interpretability when using these algorithms. It can be noted that the better the learning of an algorithm, the more difficult its interpretation is (Montavon et al., 2018).

It should be noted that the CNN model here designed is quite simple, because the most of the investment was made in the methodical aspect where we combined the robustness of Deep Learning model and the capability of OBIA for shoreline extraction, in particular rocky shoreline. Indeed. Furthermore, complex architectures can be performed.

Moreover, the combined methods were tested on a micro-tidal coast, with insignificant tidal range $(0.17 \mathrm{~m})$ (Ali, 2016). In case of shoreline detection relative to macro-tidal coast, this method would not be suitable without including an estimation of the tidal range.

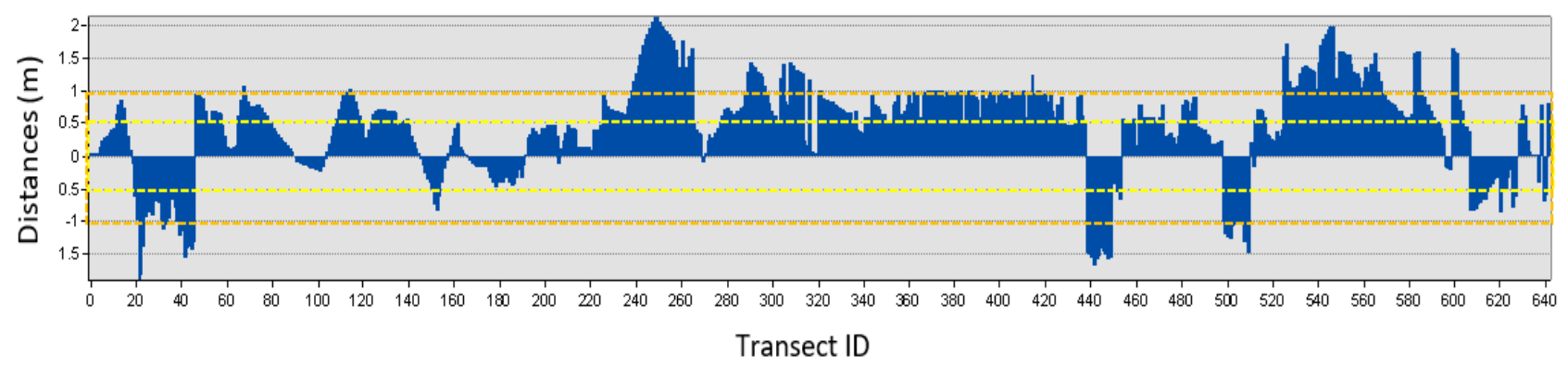

Figure 6. Distances between extracted and digitized shorelines. 


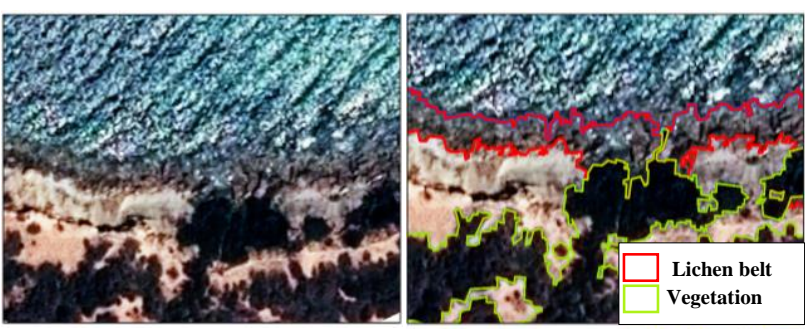

Figure 7. The misclassification and confusion between Lichen and Vegetation

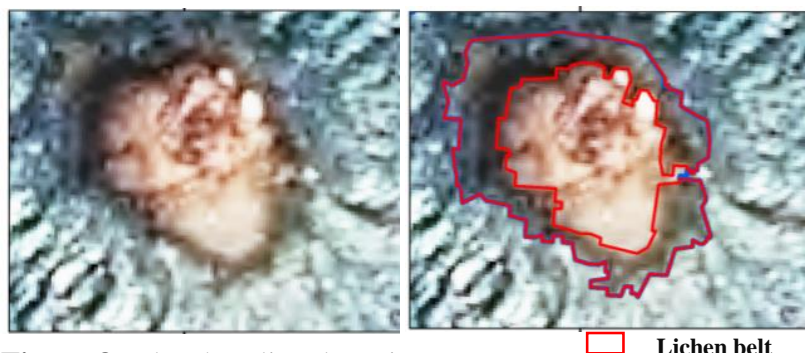

Figure 8. Islet shoreline detection

\section{CONCLUSION}

Integrating the CNN models with the OBIA capabilities has proved it efficiency for rocky shoreline extraction. The result has been compared to a reference shoreline revealing a very good accuracy.

The innovative method, here developed, can be used for a multi-date shoreline change assessment and therefore for coastal erosion studies. It can be reproduced to optimize the time/costs and reduce the subjective component of traditional methods consisting in manual digitizing or in-situ measurement.

The most of the investment of this study was made on the methodical aspect where we combined the robustness of the Deep Learning model in the classification of remotely sensed images and the capability of OBIA to detect borders for the extraction of rocky shoreline. Indeed the designed architecture of the CNN model is simple but it has proven it efficiency using multispectral images such as Pleiades, which are characterized by a very high special resolution, however further more complex architectures can be performed according to the data type and the application objectives.

In this study, CNN-OBIA methods has been tested for rocky shoreline of micro-tidal coasts in the western Mediterranean basin. Further application of these methods on other type of coasts may be unsuitable without a tidal range consideration.

\section{ACKNOWLEDGEMENTS}

This work was supported by the [Hubert Curien (PHC) Tassili] project under Grant [19 MDU 207] and PNE grants of Algerian Ministry of Higher Education and Scientific Research. The authors are grateful to GEOSUD for the Pleiades imagery, and to Centre Technique Spatial-Algeria for the GPS survey.

\section{REFERENCES}

Ali, R., 2016. Traitement du signal marégraphique par analyse harmonique pour la détermination d'une référence altimétrique pour l'Algérie. BSG 19, 2-10.

Baatz, M. and Schape, A., 2000. Multiresolution Segmentation: An Optimization Approach for High Quality Multi-Scale Image Segmentation. In: Strobl, J., Blaschke, T. and Griesbner, G., Eds., Angewandte Geographische Informations-Verarbeitung, XII, Wichmann Verlag, Karlsruhe, Germany, 12-23.

Bagli, S., Soille, P., 2003. Morphological Automatic Extraction of Pan-European Coastline from Landsat ETM + Image. Presented at the International Symposium on GIS and Computer Cartography for Coastal Management, Genova, 26 September, pp. 58-69.

Bengoufa, S, Niculescu, S Mustapha Kamel Mihoubi, Rabah Belkessa, Ali Rami, Walid Rabehi, Katia Abbad, "Machine learning and shoreline monitoring using optical satellite images: case study of the Mostaganem shoreline, Algeria," J. Appl. Remote Sens. 15(2), $026509 \quad$ (2021), doi: 10.1117/1.JRS.15.026509.Boak, E.H., Turner, I.L., 2005. Shoreline Definition and Detection: A Review. Journal of Coastal Research 21, 688-703. https://doi.org/10.2112/030071.1

Bonnot-Courtois, C., Levasseur, J-E., Denantes, S., 2013. Reconnaissance de la limite terrestre du domaine maritime : intérêt et potentialités de critères morpho-sédimentaires et botaniques.CETMEF,

http://www.bv.transports.gouv.qc.ca/mono/1159947.pdf

Davies, C.E., Moss D., Hill, M.O., 2004. EUNIS Habitat Classification, Revised 2004. Report to European Environment Agency, European Topic Centre on Nature Protection and Biodiversity. October 2004.

Campos-Taberner, M., García-Haro, F.J., Martínez, B., Izquierdo-Verdiguier, E., Atzberger, C., Camps-Valls, G., Gilabert, M.A., 2020. Understanding deep learning in land use classification based on Sentinel-2 time series. Scientific Reports 10, 17188. https://doi.org/10.1038/s41598-020-74215-5

Campos-Taberner, M., Romero-Soriano, A., Gatta, C., CampsValls, G., Lagrange, A., Le Saux, B., Beaupère, A., Boulch, A., Chan-Hon-Tong, A., Herbin, S., Randrianarivo, H., Ferecatu, M., Shimoni, M., Moser, G., Tuia, D., 2016. Processing of Extremely High-Resolution LiDAR and RGB Data: Outcome of the 2015 IEEE GRSS Data Fusion Contest-Part A: 2-D Contest. IEEE Journal of Selected Topics in Applied Earth Observations and Remote Sensing 9, 5547-5559. https://doi.org/10.1109/JSTARS.2016.2569162

García-Rubio, G., Huntley, D., Russell, P., 2015. Evaluating shoreline identification using optical satellite images. Marine Geology 359, 96-105. https://doi.org/10.1016/j.margeo.2014.11.002

Ghorbanzadeh, O., Tiede, D., Wendt, L., Sudmanns, M., Lang, S., 2021. Transferable instance segmentation of dwellings in a refugee camp - integrating CNN and OBIA. European Journal 
of Remote Sensing 54, 127-140. https://doi.org/10.1080/22797254.2020.1759456

LeCun, Y., Bengio, Y., Hinton, G., 2015. Deep learning. Nature 521, 436-444. https://doi.org/10.1038/nature14539

Liu, T., Abd-Elrahman, A., Morton, J., Wilhelm, V.L., 2018. Comparing fully convolutional networks, random forest, support vector machine, and patch-based deep convolutional neural networks for object-based wetland mapping using images from small unmanned aircraft system. GIScience \& Remote Sensing 55, 243-264. https://doi.org/10.1080/15481603.2018.1426091

Mallet C., Michot A. De La Torre Y., Lafon V., Robin M., Prevoteaux, B., 2012. Synthèse de référence des techniques de suivi du trait de côte - Rapport BRGM/RP-60616-FR, 162 p.

Montavon, G., Samek, W., Müller, K.-R., 2018. Methods for interpreting and understanding deep neural networks. Digital Signal Processing 73, 1-15. https://doi.org/10.1016/j.dsp.2017.10.011

Niculescu, S., Billey, A., Jr, H.T.-O.-A., 2018. Random forest classification using Sentinel-1 and Sentinel-2 series for vegetation monitoring in the Pays de Brest (France), in: Remote Sensing for Agriculture, Ecosystems, and Hydrology XX. Presented at the Remote Sensing for Agriculture, Ecosystems, and Hydrology XX, International Society for Optics and Photonics, p. 1078305. https://doi.org/10.1117/12.2325546

Romero, A., Gatta, C., Camps-Valls, G., 2016. Unsupervised Deep Feature Extraction for Remote Sensing Image Classification. IEEE Transactions on Geoscience and Remote Sensing 54, 1349-1362. https://doi.org/10.1109/TGRS.2015.2478379

Thieler, E.R., Himmelstoss, E.A., Zichichi, J.L., Ergul, A., 2009. The Digital Shoreline Analysis System (DSAS) Version 4.0 - An ArcGIS extension for calculating shoreline change (USGS Numbered Series No. 2008-1278), The Digital Shoreline Analysis System (DSAS) Version 4.0 - An ArcGIS extension for calculating shoreline change, Open-File Report. U.S. Geological Survey, Reston, VA. https://doi.org/10.3133/ofr20081278

Westoby, M.J., Lim, M., Hogg, M., Pound, M.J., Dunlop, L., Woodward, J., 2018. Cost-effective erosion monitoring of coastal cliffs. Coastal Engineering 138, 152-164. https://doi.org/10.1016/j.coastaleng.2018.04.008

Zhu, X.X., Tuia, D., Mou, L., Xia, G., Zhang, L., Xu, F., Fraundorfer, F., 2017. Deep Learning in Remote Sensing: A Comprehensive Review and List of Resources. IEEE Geoscience and Remote Sensing Magazine 5, 8-36. https://doi.org/10.1109/MGRS.2017.2762307 